\title{
REFLETINDO SOBRE A APRENDIZAGEM: AS TEORIAS DE JEAN PIAGET E DAVID AUSUBEL
}

Fernanda Miranda Caliani, Rebeca de Carvalho Bressa

Universidade do Oeste Paulista - UNOESTE, Mestrado em Educação, Presidente Prudente, SP. E-mail: rebecabressa@gmail.com

\section{RESUMO}

Este artigo tem como objetivo explanar e refletir os principais pontos que fundamentam as teorias de Piaget e Ausubel, suas implicações e aplicabilidade nos processos de aprendizagem. Para atingir tal objetivo, utilizamos como metodologia a pesquisa bibliográfica onde autores desta área propõem uma discussão acerca da teoria das teorias de Piaget e Ausubel. E com a contribuição desses autores podemos observar que a teoria da Aprendizagem Significativa de David Ausubel fundamentada em Jean Piaget atinge um ponto fundamental ao processo de ensino e aprendizagem, onde o aluno é o sujeito de sua aprendizagem, que depende de seus conhecimentos prévios e da interatividade com o meio, que provocam novos conhecimentos significativos para ele. O professor deve estar atento a fazer intervenções, levando em consideração a formação da estrutura cognitiva do indivíduo.

Palavras-Chave: Jean Piaget, David Ausubel, modelo epistemológico, modelo pedagógico, teorias de aprendizagem

\section{REFLECTING ON LEARNING: THE THEORIES OF JEAN PIAGET AND DAVID AUSUBEL}

\begin{abstract}
This article aims to explain and reflect the main points that underlie the theories of Piajet and Ausebel, their implications and applicability in the learning processes. In order to reach this objective, we used as methodology the bibliographical research where authors of this area propose a discussion about the theories of Piaget and Ausubel. With the contribution of these authors, we can observe that David Ausubel's Theory of Significant Learning based on Jean Piaget has a fundamental point in the process of teaching and learning, where the student is the subject of his learning, which depends on his previous knowledge and interactivity with the environment, which provoke significant new knowledge for him. The teacher should be alert to making interventions, taking into account the formation of the individual's cognitive structure.
\end{abstract}

Keywords: Jean Piaget, David Ausubel, epistemological model, pedagogical model, learning theories 


\section{INTRODUÇÃO}

O Homem tem "o saber" como uma das principais características do seu desenvolvimento. Várias ciências procuram facilitar os processos envolvidos na aprendizagem e nas relações da convivência humana. A Pedagogia surge com a função histórica de preparar o educando para a vida na sua completude. E, "a ação educativa é orientada por teorias e modelos epistemológicos, seja de maneira explícita ou implícita” (SAVARIS, LAZZARIN, TREVISOL, 2016, p. 84). Esses modelos adotados na educação estão resumidos no quadro abaixo:

Quadro 1. Modelos epistemológico pedagógico e teorias da aprendizagem.

\begin{tabular}{|c|c|c|}
\hline $\begin{array}{l}\text { Modelo } \\
\text { epistemológico }\end{array}$ & Modelo pedagógico & Teorias da aprendizagem \\
\hline $\begin{array}{c}\text { Empirismo } \\
\mathrm{S} \leftarrow \mathrm{O} \\
\text { (sujeito) (objeto) }\end{array}$ & $\begin{array}{c}\text { Pedagogia diretiva } \\
\mathrm{A} \leftarrow \mathrm{P} \\
\text { (aluno) } \quad \text { (professor) }\end{array}$ & $\begin{array}{l}\text { Teoria } \\
\text { comportamental/Behaviorista } \\
\mathrm{E} \rightarrow \mathrm{R} \rightarrow \text { Reforço } \\
\quad \text { (estímulo) (resposta) }\end{array}$ \\
\hline $\begin{array}{c}\text { Apriorismo } \\
\mathrm{S} \rightarrow \mathrm{O} \\
\text { (sujeito) (objeto) }\end{array}$ & $\begin{array}{l}\text { Pedagogia não diretiva } \\
\qquad \begin{array}{l}\mathrm{A} \rightarrow \mathrm{P} \\
\text { (aluno) } \quad \text { (professor) }\end{array}\end{array}$ & \begin{tabular}{l}
\multicolumn{2}{c}{ Gestalt / Humanista } \\
(Köhler,Rogers / Teoria \\
figura/fundo)
\end{tabular} \\
\hline $\begin{array}{c}\text { Interacionismo } \\
\mathrm{S} \rightarrow 0 \\
\leftarrow \\
\text { (sujeito) (objeto) }\end{array}$ & $\begin{array}{l}\text { Pedagogia relacional } \\
\qquad \rightarrow \mathrm{P} \\
\text { (aluno) (professor) }\end{array}$ & $\begin{array}{ll}\text { Construtivismo; } & \text { histórico- } \\
\text { cultural } \\
\text { S OS O } \\
\text { (centrada na ação) } \\
\text { na linguagem) }\end{array}$ \\
\hline
\end{tabular}

Fonte: Trevisol, 2013. Nota: As setas indicam a origem e o destino das ações.

No empirismo a filosofia do conhecimento humano está baseada na experiência humana sensorial, onde os sentidos são importantes para o "saber" do sujeito. A partir da teoria comportamental behaviorista, a aprendizagem advém da mudança no comportamento provocada por aquele que ensina e que usa estímulos reforçadores sobre aquele que aprende. Com este pensamento a pedagogia é diretiva, somente o professor ensina. No apriorismo, o sujeito traz consigo condições de conhecimento e aprendizagem desde o nascimento, que aparecerão progressivamente ao longo do processo de maturação. A ação do conhecimento é exclusiva do sujeito, o meio não tem participação. Com a teoria de aprendizagem Humanista, o conhecimento é inerente ao ser e necessita ser despertado. O professor vai auxiliar no aprendizado, é um facilitador e sua intervenção no processo de aprendizagem do aluno é inexpressiva (PERES, 2014).

Já no interacionismo, o saber será constituído pela interação entre o sujeito e o objeto, de forma recíproca. O principal teórico deste modelo é Jean Piaget, o qual desenvolveu a teoria da epistemologia genética. O sujeito se desenvolve pela sua própria ação e relação com o meio social, cultural e econômico. Deste modelo epistemológico, apresentam-se as teorias cognitivistas: verbal significativa (por David Ausubel), construtivismo (por Jean Piaget) e históricocultural (por Vygotsky) (PERES, 2014; FACIN, 2015).

O objetivo deste artigo é o de refletir sobre os principais pontos que fundamentam as teorias de Jean Piaget e David Ausubel, representantes do cognitivismo, e suas implicações com a aprendizagem. 


\section{METODOLOGIA}

Esta pesquisa foi realizada na perspectiva de um ensaio teórico (SALVADOR, 1991), com autores desta área que propõem uma discussão acerca da aprendizagem com a contribuição das teorias de Jean Piaget e David Ausubel.

\section{DISCUSSÃO}

\section{JEAN PIAGET E A TEORIA DA EPISTEMOLOGIA GENÉTICA}

De acordo com Goulart (2008) e Munari (2010), Piaget estudou o pensamento infantil e contribuiu imensamente com a Educação ao descrever sobre a psicologia das crianças e as fases do desenvolvimento infantil. Sua teoria está fundamentada na ideia que o conhecimento ocorre a partir de um processo interativo entre o sujeito e o meio, o Construtivismo. A Epistemologia Genética estuda o pensamento da criança e como ele se desenvolve até alcançar o raciocínio adulto (lógico e científico). Piaget procura entender a capacidade de conhecer e aprender da criança. O sujeito é epistêmico, busca o conhecimento. Quando em equilíbrio com o meio está em adaptação e quando está frente a situações - problemas, entra em desequilíbrio e desadaptação com o ambiente, desencadeando um conflito cognitivo.

Para Piaget, o indivíduo retorna ao estado de equilíbrio e adaptação naturalmente, através de dois mecanismos cognitivos: a assimilação e a acomodação. A assimilação é a tentativa de solucionar o problema relacionando há um conhecimento já presente em suas estruturas cognitivas. $\mathrm{Na}$ acomodação o indivíduo modifica as estruturas cognitivas antigas, constrói novas associações ao conhecimento prévio, domina a situação e amplia a cognição sobre a situaçãoproblema com a nova informação. Durante o desenvolvimento a criança passa por etapas nas quais ela terá uma determinada organização mental (estado de equilíbrio) que será modificada à medida que adquire novas informações e nova forma de compreender a realidade. Quando chegar ao final da adolescência o indivíduo terá a maneira intelectual pronta para a vida adulta (GOULART, 2008; MUNARI, 2010; PIAGET, 2013).

$A$ avaliação da inteligência para Piaget tem uma abordagem Psicogenética, onde o investigador deve querer compreender o que leva o sujeito a ter esta resposta especifica. A resposta tem um controle psicológico e não uma padronização como é o controle fisicalista (GOULART, 2008; MUNARI, 2010; PIAGET, 2013). “A inteligência aparece como uma estruturação que imprime determinadas formas aos intercâmbios entre o sujeito ou sujeitos e os objetos circundantes, próximos ou mais afastados" (PIAGET, 2013, p.235).

Os Estádios de Desenvolvimento Cognitivo foram descritos na Teoria Epistemologia Genética e se caracterizam por períodos, que seguem uma mesma sequência, nos quais a criança consegue estado de equilibração por uma fase de preparação e outra de acabamento. Os estádios são integrados gradativamente e no final os esquemas construídos são usados pelo indivíduo de forma interdependente. Estádio Sensório-Motor ( 0 a 2 anos), o bebê apresenta ações reflexas como sucção e preensão. No início é passivo e tem apenas esquemas que funcionam de maneira repetitiva como pegar, olhar, bater. Com o tempo irá desenvolver comportamentos conscientes e terá domínio de algumas ações. Esse processo envolve a: Assimilação Funcional, Generalizada e Recognitiva (GOULART, 2008; MUNARI, 2010; PIAGET, 2013).

A Funcional caracteriza-se pela repetição do esquema. A Generalizada caracterizase pela utilização do esquema em diferentes situações e na Recognitiva há um reconhecimento dos esquemas. Apresenta seis sub-estádios. 1․ (0 a 1 mês): formação dos primeiros processos cognitivos por atividades reflexas (preensão e sucção). 2‥ (1 a 4 meses): reação circular primária, repete ações reflexas em função da experiência, da adaptação adquirida. 3‥ (4 a 8 meses): reação circular secundária, deseja repetir resultados inusitados para obter um objeto ou manter espetáculos interessantes. 4ㅇ. (8 a 12 meses): aplica meios já conhecidos para solucionar situações novas, já é capaz de coordenar, generalizar diferentes ações para atingir objetivos, mas sem 
planejamento. 5․ (12 a 18 meses): reação circular terciária, inventa novas forma de agir para atingir um fim, tem maior diversificação e controle de ações. 60. (18 a 24 meses): período de transição, vai iniciar a fase da representação, com a imaginação de acontecimentos, combinação de ações mentais ao invés de apenas físicas e surgimento da linguagem (GOULART, 2008; MUNARI, 2010; PIAGET, 2013).

O Estádio Pré-operatório ( 2 a 6 anos) é caracterizado pelo desenvolvimento da inteligência representativa. A função simbólica possibilita que a criança represente um objeto ausente (significante) por meio de um significado. Este estádio pré-lógico é marcado por várias características: pensamento egocêntrico, transdutivo, justaposição, sincretismo, centração, pensamento artificialista e finalista, pensamento animista, provas operatórias e relacionamento social (GOULART, 2008; MUNARI, 2010; PIAGET, 2013).

No Estádio Operatório Concreto (7 a 11 anos) o pensamento passa a ser operatório. A criança começa a coordenar pontos de vista de forma lógica e expressa ação cognitiva elaborada. Mas ainda precisa da observação de objetos concretos. É marcado por vários fatores: pensamento indutivo, lógico, relacionamento social, o qual tem progresso significativo, pois diminui o egocentrismo e desenvolve da linguagem socializada (GOULART, 2008; MUNARI, 2010; PIAGET, 2013).

No último estádio, o Estádio Operatório Formal (12 a 15 anos), o adolescente consegue formar esquemas conceituais abstratos (amor, saudade, justiça) e realizar operações mentais com lógica formal, mais elaboradas em conteúdo e raciocínio. A partir desse estádio o pensamento é hipotético-dedutivo. O sujeito consegue discutir sobre valores morais, constrói seus próprios valores, adquire autonomia, levanta e propõem suas hipóteses e faz julgamentos com justificativas apropriadas. Nessa fase o indivíduo forma sua identidade e a lógica antes indutiva passa a ser dedutiva, na qual o raciocínio é mais sofisticado e o pensamento abstrato. Conforme Piaget agora o sujeito atingiu a forma definitiva de equilíbrio e complexa do pensamento, permitindo a compreensão de teorias cientificas (GOULART, 2008; MUNARI, 2010; PIAGET, 2013).

\section{DAVID AUSUBEL E A TEORIA DA APRENDIZAGEM SIGNIFICATIVA}

Ausubel contribuiu para a Educação com a Teoria da Aprendizagem Significativa, na qual procura explicar os processos de aprendizagem humana, embasado nos princípios organizacionais da cognição. Valoriza o conhecimento e o entendimento das informações. Para Ausubel, a "aprendizagem significativa é o processo através do qual uma nova informação (um novo conhecimento) se relaciona de maneira não arbitrária e substantiva (não-literal) à estrutura cognitiva do aprendiz" (MOREIRA, 2011, p. 26).

A não arbitrariedade é a forma como os conhecimentos prévios são resgatados. Estes conhecimentos são denominados de "subsunçores" e servem de âncora para a aquisição de novos conceitos. O novo conhecimento é não-literal porque o que deve ser incorporado à cognição é a substância deste conhecimento, das novas ideias e não as palavras precisamente ditas. Assim um mesmo conceito pode ser expresso de diferentes formas (AUSUBEL, 2003).

Duas condições são necessárias para haver aprendizagem significativa. $\mathrm{O}$ aprendiz deve ter disposição para aprender e o conteúdo a ser aprendido tem que ser lógico e psicológico, ou seja, ter uma experiência pessoal (PELIZZARI et al, 2002). A estrutura cognitiva se organiza em níveis: abstração, generalidade e inclusividade de conteúdo (MOREIRA, 2011). E a repetição para aprender deve ser substituída pelo auto estruturação significativa. Também é necessário que os "subsunçores" se relacionem, ou seja, façam a relação entre ideias e conceitos. Essa combinação de dados é conhecida como reconciliação integrativa (MOREIRA, 1998; AUSUBEL, 2003).

Construir um currículo baseada na teoria de Ausubel requer: identificar a estrutura de significados da matéria; identificar "subsunçores" do assunto; identificar conhecimento prévio 
no cognitivo do aprendiz; organizar o conteúdo, usando ideias de diferenciação progressiva e reconciliação integrativa e ensinar usando organizadores prévios (MOREIRA, 1998).

Para Ausubel, professor e aluno devem evitar a aprendizagem mecânica pura. Esta aprendizagem não se torna significativa, pois não teve relações e ligações na estrutura cognitiva. Fica apenas na memorização. Os conceitos são armazenados de forma arbitraria, não-substantiva, sem garantir flexibilidade no seu uso, nem longevidade. Já a aprendizagem significativa ocorre por meio da descoberta ou da recepção. Na descoberta o aluno aprende sozinho, semelhante a resolução de um determinado problema. Na recepção a informação está pronta (aula expositiva) e o aluno deve ativamente relacionar o assunto as ideias relevantes já presentes na sua estrutura cognitiva. A modalidade por recepção pode ser representacional, de conceitos ou de proposições (MOREIRA, MASINI, 2001; AUSUBEL, 2003).

A Aprendizagem Significativa Representacional é a mais próxima da mecânica, ocorre quando um símbolo arbitrário adquire o significado equivalente ao seu referente. De acordo com a Teoria: "A aprendizagem representacional (tal como a atribuição de um nome) aproxima-se da aprendizagem por memorização" (AUSUBEL, 2003, p.1). Ela será significativa na medida que as proporções de equivalência representacional se relacionam, de forma nãoarbitrária, a uma condição existente na cognição da pessoa (AUSUBEL, 2003).

Aprendizagem Significativa por Conceitos consiste em relacionar os conceitos de forma abstrata e diversificada, podendo atribuir muita complexidade a um assunto especifico, relacionado de várias formas na estrutura cognitiva. "Podem definir-se os conceitos como objetos, acontecimentos, situações ou propriedades que possuem atributos específicos comuns e são designados pelo mesmo signo ou símbolo" (AUSUBEL, 2003, p.2). Acontece de duas formas: formação de conceitos (prevalente na infância) e assimilação de conceitos (forma dominante na escola). Na formação de conceitos, muitos dos atributos do conceito são adquiridos por experiência direta. Ainda existe a definição de conceitos superordenados e subordinados. Os superordenados são mais complexos e ancoradouro para novas ideias, podendo classificar os novos atributos que estão sendo aprendidos. Os subordinados podem ser classificados por outro superordenado já aprendido ou não (MOREIRA, MASINI, 2001; AUSUBEL, 2003).

$\mathrm{Na}$ Aprendizagem Significativa por Proposições haverá mais de um conceito envolvido. Semanticamente um conceito é mais inclusivo que o outro. E há uma forma particular de combinar esses conceitos. Ela é semelhante a representacional e, na medida que surgem novos conceitos depois de uma tarefa "será significativa se relacionar e interagir com ideias relevantes existentes na estrutura cognitiva" (AUSUBEL, 2003, p.2). "O conteúdo cognitivo distinto é um produto interativo do modo particular como o conteúdo da nova proposição está relacionado com o conteúdo de ideias estabelecidas e relevantes existentes na estrutura cognitiva" (AUSUBEL, 2003, p. 3).

A Aprendizagem Significativa por Proporções pode acontecer de forma subordinada, superordenada e combinatória. A subordinada está vinculada a uma ideia superordenada e, portanto, a nova proposição exemplifica um conceito já existente na estrutura cognitiva (chamada derivada) ou a nova proposição amplia, elabora, modifica ou qualifica ideias previamente aprendidas. A superordenada acontece quando há relação de ideias subordinadas especificas ou coleção geral de conceitos relevantes que possam ser incluídos na estrutura cognitiva. Já a Aprendizagem Significativa por Proporções combinatória descreve a situação na qual uma proposição com potencial significativo não pode ser relacionada a conceitos relevantes a cognição do estudante, mas acaba sendo relacionável a uma combinação de ideias com relevância variada (MOREIRA, MASINI, 2001; AUSUBEL, 2003). 


\section{TEORIAS DE PIAGET E AUSUBEL: IMPLICAÇÕES COM A APRENDIZAGEM}

A contribuição dessas duas teorias foi expressiva para a educação. Esses teóricos trouxeram informações imprescindíveis sobre o entendimento da aprendizagem. Piaget favoreceu o desenvolvimento infantil, mas também trouxe informações sobre a aprendizagem funcional do adulto. Ausubel focou no processo de aprendizagem sem direcionar a idade, favorecendo e aprofundando as informações sobre o processo de assimilação explicado por Piaget. A aprendizagem subordinada derivativa de Ausubel tem proximidade com o conceito de assimilação de Piaget. Os dois concordam que assimilar é incorporar um novo conceito em um padrão já existente na estrutura cognitiva e que a aprendizagem é focada no aluno. O professor é um mediador importante, mas a participação do estudante deve ser ativa para que haja efetividade no aprendizado (PONTES NETO, 2006).

O erro do estudante toma uma nova dimensão após essas teorias, pois ele permite compreender as ações intelectuais da criança e do estudante e contribui para relacionar novos conceitos de forma significativa. O processo de acomodação descrito por Piaget tem respaldo nos conceitos de aprendizagem superordenada. Embora haja várias concordâncias sobre o desenvolvimento cognitivo e sobre a importância da motivação intrínseca do indivíduo, seus trabalhos são distintos. Ausubel não descreveu qualquer influência de Piaget em sua obra (PONTES NETO, 2006).

Ausubel concorda que pode haver semelhança com a noção de assimilação, pois Piaget permite a relação com esquemas já existentes. Mas refere que sua teoria aprofunda este conhecimento, pois descreve explicitamente o processo de assimilação e sua progressão evolutiva ao invés de somente fenômeno.

\section{CONCLUSÃO}

A teoria da Aprendizagem Significativa de David Ausubel fundamentada em Jean Piaget atinge um ponto fundamental ao processo de ensino/aprendizagem, onde o aluno é o sujeito de sua aprendizagem, que depende de seus conhecimentos prévios e da interatividade com o meio, que provocam novos conhecimentos significativos para ele. Portanto, o professor deve estar atento a fazer intervenções, levando em consideração a formação da estrutura cognitiva do indivíduo.

\section{REFERÊNCIAS}

AUSUBEL, D. P. Aquisição e Retenção de Conhecimentos: Uma Perspectiva Cognitiva. Lisboa: Plátano Edições Técnicas, 2003.

FACIN, E. C. Reflexões sobre os modelos epistemológicos e pedagógicos de um grupo de educadores. Unoesc \& Ciência - ACHS, Joaçaba, v. 6, n. 1, p. 99-110, jan./jun. 2015.

GOMES, A.P. et al. A Educação Médica entre mapas e âncoras: a aprendizagem significativa de David Ausubel, em busca da Arca Perdida. Revista Brasileira de Educação Médica, 32 (1): 105 111; 2008, https://doi.org/10.1590/S0100-55022008000100014.

GOULART, I. B. Piaget: experiências básicas para utilização pelo professor. 24a ed. rev. Ed. Petrópolis, RJ: Vozes, 2008.

MOREIRA. M.A. Mapas conceituais e aprendizagem significativa. Cadernos de Aplicação, 11(2): 143-156, 1998. 
MOREIRA, M.A. Aprendizagem significativa: um conceito subjacente. Aprendizagem Significativa em Revista, v.1(3), p. 25-46, 2011.

MOREIRA, M.A, MASINI, E.F.S. Aprendizagem significativa: a Teoria de David Ausubel. São Paulo: Centauro, 2001.

MUNARI, A. Jean Piaget. Recife: Fundação Joaquim Nabuco, Editora Massangana, 2010.

PELIZZARI, A. et al. Teoria da aprendizagem significativa segundo Ausubel. Rev. PEC, Curitiba, v.2, n.1, p.37-42, jul. 2001-jul. 2002.

PERES, C.M. et al. Abordagens pedagógicas e sua relação com as teorias de aprendizagem. Medicina (Ribeirão Preto) 47(3): 249-55; 2014.

PIAGET, J. A Psicologia da Inteligência. Petrópolis, RJ: Vozes, 2013.

PONTES NETO, J.A. da S. Teoria da aprendizagem significativa de David Ausubel: perguntas e respostas. Série-Estudos - Periódico do Mestrado em Educação da UCDB, Campo Grande - MS, n. 21, p.117-130, jan./jun. 2006.

SALVADOR, A.D. Métodos e técnicas de pesquisa bibliográfica. Porto Alegre: Sulina Editora, 1991.

SAVARIS, L, LAZZARIN, M. S. B, TREVISOL, M. T.C. Teoria e prática docente: aproximações ou distanciamentos? Cadernos de Pesquisa: Pensamento Educacional, Curitiba, v. 11, n. 28, p.83-108 maio/ago. 2016.

TREVISOL, M. T. C. Teoria e prática educativa. Joaçaba: Unoesc, 2013. 